\title{
Irradiation, Cisplatin, and 5-Azacytidine Upregulate Cytomegalovirus Promoter in Tumors and Muscles: Implementation of Non-invasive Fluorescence Imaging
}

\author{
Urska Kamensek, Gregor Sersa, Suzana Vidic, Gregor Tevz, Simona Kranjc, \\ Maja Cemazar
}

Department of Experimental Oncology, Institute of Oncology Ljubljana, Zaloska 2, SI-1000, Ljubljana, Slovenia

\begin{abstract}
Purpose: The cytomegalovirus (CMV) promoter is one of the most commonly used promoters for expression of transgenes in mammalian cells. The aim of our study was to evaluate the role of methylation and upregulation of the CMV promoter by irradiation and the chemotherapeutic agent cisplatin in vivo using non-invasive fluorescence in vivo imaging.

Procedures: Murine fibrosarcoma LPB and mammary carcinoma TS/A cells were stably transfected with plasmids encoding CMV and p21 promoter-driven green fluorescent protein (GFP) gene. Solid TS/A tumors were induced by subcutaneous injection of fluorescent tumor cells, while leg muscles were transiently transfected with plasmid encoding GFP under the control of the CMV promoter. Cells, tumors, and legs were treated either by DNA methylation inhibitor 5-azacytidine, irradiation, or cisplatin. GFP expression was determined using a fluorescence microplate reader in vitro and by non-invasive fluorescence imaging in vivo.

Results: Treatment of cells, tumors, and legs with 5-azacytidine (re)activated the CMV promoter. Furthermore, treatment with irradiation or cisplatin resulted in significant upregulation of GFP expression both in vitro and in vivo.

Conclusions: Observed alterations in the activity of the CMV promoter limit the usefulness of this widely used promoter as a constitutive promoter. On the other hand, inducibility of CMV promoters can be beneficially used in gene therapy when combined with standard cancer treatment, such as radiotherapy and chemotherapy.
\end{abstract}

Key words: CMV promoter, Fluorescence imaging, Irradiation, Cisplatin, Demethylation, In vivo, Mice, Mammary carcinoma, Fibrosarcoma

\section{Introduction}

Tmmediate/early promoter/enhancer of cytomegalovirus 1 (CMV promoter) is one of the most commonly used promoters for expression of transgenes in mammalian cells for research or therapeutic purposes. Although it is often

Correspondence to: Maja Cemazar; e-mail: mcemazar@onko-i.si thought of as a constitutive and unregulated pan-specific promoter, it has been shown that its activity is strongly dependent on the host-cell transcriptional environment. Many reports demonstrated that it does not direct persistent transgene expression and that its transcriptional activity varies according to cell type and developmental age and can also be upregulated under specific conditions [1-13].

DNA methylation of the promoter is the most frequently given explanation for transient gene expression of the 
transgene constructs containing the CMV promoter, independently of the delivery system used [1-5]. For instance, the CMV promoter was shown to be susceptible to transcriptional inactivation by methylation after transient transfection using different non-viral delivery vectors [2, 4, 5]. The same observation was also demonstrated for a stably transfected cell line using lipofection in vitro [3]. Inhibition of transgene expression caused by methylation of the CMV promoter was also reported in vivo following adenoviral gene delivery to muscle [1]. On the other hand, some studies demonstrated that methylation is not always responsible for the observed transient expression in vivo. For instance, methylation was investigated as a potential cause of the transient transgene expression from non-viral vectors in the mouse lung, but results suggested that the plasmid DNA does not become de novo methylated in the mouse airways [6]. Similarly, transcriptional inactivation of CMV after adenovirus gene transfer was demonstrated in the mouse liver although there was no evidence for the methylation of adenovirus DNA [7]. In the later study [7], silenced CMV promoter was reactivated after treatment with lipopolysaccharide (LPS). There have also been other reports suggesting that the CMV promoter can be upregulated under specific conditions such as treatment with LPS, cyclic AMP, phorbol esters, bacterial CpGs [8-11], and by a variety of environmental stresses [12]. Recently, chemotherapeutic agents and radiation (IR) were shown to upregulate expression of transgenes driven by the CMV promoter in vitro and in vivo [13, 14]. Upregulation with chemotherapeutics was reported to occur in multiple cell lines independently of the transfection method used [14], and radiation was reported to enhance adenoviral gene therapy in pancreatic cancer via activation of the CMV promoter [13].

Non-invasive whole body optical methods (luminescence or fluorescence) are very competitive approaches to assess tumor development and responses to drug and gene therapies in vivo and are far more affordable compared to other imaging methods, such as positron emission tomography, Xray computed tomography, and magnetic resonance imaging $[15,16]$. Recently, they were employed also for following transgene expression and activity of promoters in vivo. However, the in vivo reports on methylation and upregulation of the CMV promoter by conventional treatments, such as IR and cisplatin (CDDP), are scarce; and results are contradictory $[1,6,7,14,17]$. Therefore, the aim of our study was to evaluate the role of methylation and upregulation of the CMV promoter by IR and CDDP in vivo using non-invasive fluorescence in vivo imaging, which enables long-term follow-up of reporter gene fluorescence in the animals and consequently, the activity of promoters that control reporter gene expression. Different systems in which expression of the reporter gene was controlled either by an inducible promoter or CMV promoter (stable cell lines, stably transfected experimental tumors, and transiently transfected muscles) were set up using the non-viral delivery system of electroporation and the influence of treatment with 5-azacytidine, IR, and CDDP on the activity of the CMV promoter in vitro and in vivo was assessed.

\section{Materials and Methods}

\section{Plasmids}

A plasmid encoding green fluorescence protein (GFP) under the control of the CMV promoter and neomycin resistance gene (pEGFP-N1, Clontech, Basingstoke, UK) and a plasmid encoding GFP under the control of the human p21 promoter and neomycin resistance gene (p21-EGFP, kind gift from Irena Hreljac, National Institute of Biology, Ljubljana, Slovenia) were used. Human p21 promoter is an inducible mammalian promoter that is induced by DNA damage $[18,19]$. In the study, it was used as a control for the CMV promoter.

\section{Cell Lines}

Murine adenocarcinoma of the mammary glands TS/A [20] and murine fibrosarcoma LPB [21] cell lines were maintained in Eagle's minimum essential medium (EMEM, Sigma, Taufkirchen, Germany), supplemented with $10 \%$ fetal calf serum (Sigma, Taufkirchen, Germany), $2 \mathrm{mM}$ L-glutamine, $1 \mathrm{mM}$ sodium pyruvate, and $100 \mathrm{IU} / \mathrm{ml}$ penicillin/streptomycin (Pliva, Zagreb, Croatia) in a $5 \% \mathrm{CO}_{2}$ humidified incubator at $37^{\circ} \mathrm{C}$.

\section{Preparation of Stable Cell Lines Carrying CMV or p21 Promoter-Driven Reporter Gene Constructs}

LPB and TS/A cells were transfected with the pEGFP-N1 plasmid and the LPB cell line was transfected with the p21-EGFP plasmid. Electroporation was used for introduction of plasmid DNA into cells. Specifically, cells grown as a monolayer were harvested and a $2.5 \times 10^{7}$ cells $/ \mathrm{ml}$ cell suspension was prepared in electroporation buffer (125 mM sucrose, $10 \mathrm{mM} \mathrm{K}_{2} \mathrm{HPO}_{4}, 2.5 \mathrm{mM} \mathrm{KH}_{2} \mathrm{PO}_{4}, 2 \mathrm{mM}$ $\mathrm{MgCl}_{2} \times 6 \mathrm{H}_{2} \mathrm{O}$ ). A dense cell suspension with a concentration of $1 \times 10^{6}$ cells and $10 \mu \mathrm{g}$ of pEGFP-N1 in $50 \mu \mathrm{l}$ of electroporation buffer was placed between two flat parallel stainless steel electrodes with a $2 \mathrm{~mm}$ gap connected to the GT-1 electroporator (University of Ljubljana, Faculty of Electrical Engineering, Ljubljana, Slovenia) and subjected to eight square-wave electric pulses with an amplitude per distance ratio $700 \mathrm{~V} / \mathrm{cm}, 5 \mathrm{~ms}$ duration time, and $1 \mathrm{~Hz}$ repetition frequency. After electroporation cells were incubated for $5 \mathrm{~min}$ at room temperature, plated into Petri dishes, and then cultured for 2 months under increasing concentrations $(500-1,000 \mu \mathrm{g} / \mathrm{ml}$ for $\mathrm{LPB}$ cells and 1,200 $2,000 \mu \mathrm{g} / \mathrm{ml}$ for $\mathrm{TS} / \mathrm{A}$ cells) of the selection agent geneticin (Gibco Invitrogen, San Diego, CA, USA) to obtain resistant clones. Clones with the highest GFP expression were identified by fluorescence microscopy (Olympus, Hamburg, Germany), isolated, propagated, and frozen in liquid nitrogen for subsequent experiments. To determine the number of fluorescent cells, flow cytometry analysis of stable cell lines carrying CMV promoterdriven reporter gene constructs was performed: cells were trypsinized, collected, and $2 \times 10^{4}$ cells from each stable line were analyzed using flow cytometry (Becton Dickinson, Calibur, Franklin Lakes, NJ, USA). The percentage of cells stably expressing GFP was determined from the histograms. 


\section{Study Design In Vitro}

To test the influence of DNA methylation and treatment with IR or CDDP on CMV activity in in vitro conditions, cells were treated with the DNA methylation inhibitor 5-azacytidine (5-aza-2dC, Sigma) and exposed to IR and CDDP (cis-diamminedichloroplatinum (II), Pharmacia \& Upjohn S.p.A, Milan, Italy). For the methylation tests, cells were first passaged for 1 month to allow methylation of DNA to occur and then (at the sixth passage) treated with a DNA methylation inhibitor, while for the IR and CDDP exposure freshly thawed (first passage) cells were used. The treatment protocols were as follows:

- 5-aza-2dC: Cells were plated at a density of $1.5 \times 10^{4}$ cells $/ \mathrm{cm}^{2}$ and treated with 5 -aza-2dC for 3 days. A stock solution of 5-aza-2dC was prepared in phosphate buffered saline (PBS) and was freshly diluted to a working concentration of $1 \mu \mathrm{M}$ in EMEM each day of the experiment.

- IR: Cells were plated at a density of $1.7 \times 10^{4}$ cells $/ \mathrm{cm}^{2}$ and irradiated with a dose of 6 Gy using an X-ray unit Drapac 2000 (Gulmay Medical Ltd, Shepperton, UK) operating at $220 \mathrm{kV}, 10 \mathrm{~mA}$, and with $0.55 \mathrm{~mm}$ $\mathrm{Cu}$ and $1.8 \mathrm{~mm} \mathrm{Al}$ filtration.

- $C D D P$ : CDDP in the form of crystalline powder was dissolved in sterile $\mathrm{H}_{2} \mathrm{O}$ at a stock concentration of $2 \mathrm{mg} / \mathrm{ml}$. Cells were plated at a density of $2.0 \times 10^{4}$ cells $/ \mathrm{cm}^{2}$ and the stock solution of CDDP was added to EMEM to get a working concentration of $3 \mu \mathrm{g} / \mathrm{ml}$.

Three days after the treatments, cells were trypsinized, collected, and $2 \times 10^{5}$ cells were plated in 96-well microplates. Expression of the reporter gene was determined using the fluorescence microplate reader Infinite 200 (Tecan, Männedorf, Switzerland) and fluorescence microscopy (Olympus, Hamburg, Germany)

\section{Experimental Animals}

For the in vivo experiments, female BALB/c and female C57BL/6 mice obtained from the Institute of Pathology, Faculty of Medicine, University of Ljubljana, Slovenia were used. At the beginning of the experiments, the animals were 10-12 weeks old. Mice were housed and maintained in a specific pathogen-free animal colony at constant room temperature $\left(21^{\circ} \mathrm{C}\right)$ and $12 \mathrm{~h}$ light/dark cycle. Food and water were provided ad libitum. Animals were subjected to an adaptation period of 7-10 days before the experiments were carried out. All procedures on animals were performed in accordance with the official guidelines of the Ministry of Agriculture, Forestry, and Food of the Republic of Slovenia (permission no. 323-02-632/2005/6).

\section{Induction of Solid Subcutaneous Tumors}

Viable TS/A EGFP tumor cells $\left(2 \times 10^{6}\right)$ prepared from cell culture in vitro were injected dorsolaterally in $\mathrm{BALB} / \mathrm{c}$ mice. When the tumors reached approximately $40 \mathrm{~mm}^{3}$ in volume (7-10 days), mice were randomly divided into experimental groups and subjected to a specific experimental protocol.

\section{Preparation of Transiently Transfected Muscle Model Carrying CMV Promoter-Driven Reporter Gene Construct}

$\mathrm{C} 57 \mathrm{~B} 1 / 6$ mice were anesthetized with isofluran (Torrex Chiesi $\mathrm{GmbH}$, Wien, Austria) using an isoflurane vaporizer (Datex Ohmeda, Helsinki, Finland). Plasmid pEGFP-N1 $(20 \mu \mathrm{g}$ in $20 \mu \mathrm{l}$ of water) was injected into both $m$. tibialis cranialis with a thin $(26 \mathrm{G})$ needle. The hind legs were placed between two flat parallel stainless steel electrodes with rounded corners (dimensions $20 \mathrm{~mm} \times 10 \mathrm{~mm}$ ) with a $6 \mathrm{~mm}$ gap between the electrodes con- nected to the electric pulse generator Cliniporator ${ }^{\mathrm{TM}}$ (IGEA s.r.l., Carpi, Italy) and subjected to one high-voltage square-wave electric pulse with an amplitude per distance $600 \mathrm{~V} / \mathrm{cm}$ and $100 \mu$ s duration and four low-voltage square-wave electric pulses with an amplitude per distance $80 \mathrm{~V} / \mathrm{cm}, 100 \mathrm{~ms}$ duration, and $1 \mathrm{~Hz}$ repetition frequency [22]. Good contact between the electrodes and legs was assured by hair removal using hair removal cream (Vitaskin, Krka, d.d., Novo mesto, Slovenia) and use of a conductive gel (Kameleon d.o.o., Maribor, Slovenia). When the fluorescence intensity declined to $\sim 50 \%$ of the highest level [23] (approximately 4 weeks after transfection), mice were randomly divided into experimental groups and subjected to a specific experimental protocol.

\section{Study Design In Vivo}

To test the influence of DNA methylation and treatment with IR and $\mathrm{CDDP}$ on $\mathrm{CMV}$ activity in vivo, tumor-bearing $\mathrm{BALB} / \mathrm{c}$ mice and $\mathrm{C} 57 \mathrm{BL} / 6$ mice with transiently transfected muscles were treated with either 5-azacytidine, IR, or CDDP. The treatment protocols were as follows:

- 5-aza-2dC: Mice were treated twice by intraperitoneal injection of $0.2 \mathrm{mg} / \mathrm{kg}$ of 5 -azacytidine in $300 \mu \mathrm{l}$ of PBS on two consecutive days. A stock solution of 5-aza-2dC was freshly diluted to the working concentration each day of the experiment.

- IR: Tumors and transfected muscles were irradiated with a dose of 6 Gy using the same X-ray unit as used for the in vitro experiments. During irradiation, mice were restrained in special lead holders with apertures for irradiation of the tumors/legs, exposing only the tumors/ legs and shielding the rest of the body from irradiation.

- $C D D P$ : Mice were injected intravenously with $8 \mathrm{mg} / \mathrm{kg}$ of cisplatin in $100 \mu \mathrm{l}$ of sterile $\mathrm{H}_{2} \mathrm{O}$. For each experiment, a fresh stock solution was prepared from crystalline powder and diluted to the working concentration.

\section{In Vivo Non-Invasive Fluorescence Imaging}

After the treatments, fluorescence intensity of the tumors and muscles expressing GFP was followed transcutaneously using a fluorescence stereo microscope, which enabled non-invasive follow-up of the intensity and duration of GFP expression. For each observation under the microscope, hair over the tumor or muscle was removed using an electric shaver and/or hair removal cream and animals were anesthetized with isoflurane as described above.

Digital images of fluorescence were recorded everyday posttreatment for 8 days for tumors and every 2-3 days for 12 days in the case of the muscle with a digital color camera (Axiocam MRc5, Zeiss, Jena, Germany) connected to the fluorescence stereo microscope (Lumar.V12, Zeiss, Jena, Germany). During capture, tumors or legs were placed in a special holder to minimize the movement of animals caused by breathing and to ensure the same positioning at each observation.

Images were analyzed using the ImageJ software tool (National Institute of Mental Health, Research Services Branch, Bethesda, MA, USA). Images of the same tumor (Fig. 1) or muscle (Fig. 2) taken at different time points were stacked together, and the fluorescence intensity was determined by adjusting the threshold values for each stack. Adjusted mean fluorescence intensity of each 
tumor or muscle picture in the stack was then normalized to the mean fluorescence intensity at day 0 .

\section{Statistical Analysis}

The data were tested for normality of distribution using the Kolmogorov-Smirnov test. Differences between experimental groups were statistically evaluated by the Student's $t$ test. A $P$ value of less than 0.05 was considered to be statistically significant. Statistical analysis was performed using SigmaStat software (Systat Software Inc., San Jose, CA, USA).

\section{Results}

\section{Stably Transfected Cell Lines}

LPB and TS/A stable lines expressing the GFP reporter gene under the control of the CMV promoter were prepared and designated as LPB EGFP and TS/A EGFP, and the LPB cell line expressing GFP under the control of the $\mathrm{p} 21$ promoter was prepared and designated as LPB p21-EGFP. Flow cytometry analysis showed that $70 \%$ of cells stably expressed GFP in the TS/A EGFP cell line and only $20 \%$ in the LPB EGFP cell line, whereas in the LPB p21-EGFP cell line almost all cells expressed GFP (93\%; Fig. 3).

\section{Influence of DNA Methylation on CMV Inactivation In Vitro}

Cells were first passaged for 1 month (six passages) to allow methylation of DNA to occur. During this time, fluorescence intensity decreased by a factor of 2.8 in TS/A EGFP and by 1.2 in LPB EGFP, but not in LPB p21-EGFP cells. After treatment with 5 -aza-2dC, a statistically significant increase in fluorescence intensity was obtained in TS/A EGFP and LPB EGFP cell lines by a factor of 6.9 and 1.4 compared to the control (sixth passage), respectively. The increase in the LPB p21-EGFP cell line was by a factor of 1.3 (Fig. 4).

\section{IR- and CDDP-Induced Regulation of the CMV Promoter}

Exposure of cells to IR or CDDP resulted in significant upregulation of expression in both cell lines with the CMV promoter, although the effects were more evident in the TS/ A EGFP cell line. After IR, fluorescence intensity increased 1.82-fold in TS/A EGFP and 1.05-fold in LPB EGFP cells compared to the control. After treatment with CDDP, the increase in fluorescence intensity was comparable to that after IR and was 1.73-fold in the TS/A EGFP cell line and 1.03-fold in the LPB EGFP cell line. In the control group with the inducible p 21 promoter the induction was by a factor of 1.29 after IR and 1.81 after CDDP compared to the control (Fig. 5).

\section{Influence of DNA Methylation and Treatment with IR and CDDP on CMV Activity In Vivo}

Treatment of tumors 7-10 days post-induction with 5-aza$2 \mathrm{dC}$ did not significantly increase fluorescence intensity of TS/A EGFP tumors during the observation period. Only a trend of increased fluorescence intensity towards the end of the observation period was observed. However, in the transiently transfected muscles, treatment with 5-aza-2dC 4 weeks post-transfection resulted in significantly increased fluorescence intensity towards the end of the observation period on day 12 by a factor of 1.40 (Fig. 6).

Treatment with IR or CDDP resulted in significant upregulation of GFP expression in both in vivo models $(p<$ 0.05 , vs. control). After IR, the fluorescence intensity of TS/ A EGFP tumors was significantly increased by a factor of 1.26 already on the third day after treatment and by a factor of 1.24 on the fourth day. Similarly, the increase in fluorescence intensity of transiently transfected muscles following IR was by a factor of 1.17 on the second day and by a factor of 1.25 on the fifth day post-treatment. Systemic treatment with CDDP upregulated the CMV promoter, resulting in significantly increased fluorescence intensity in both TS/A EGFP tumors and transiently transfected muscles. Upregulation was the most pronounced on
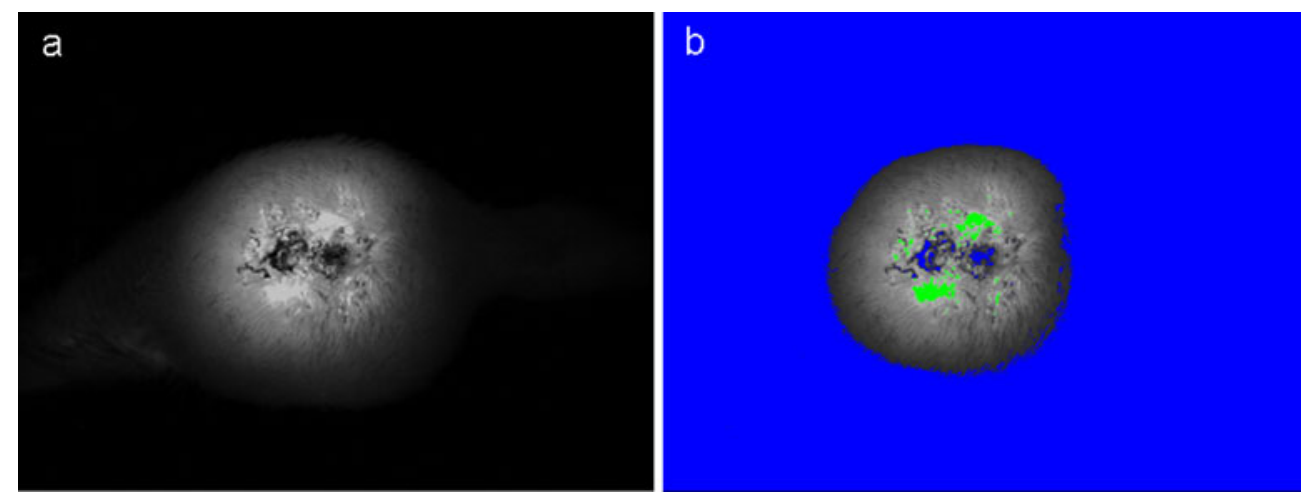

Fig. 1. a Fluorescent image of the tumor. b Fluorescence of tumors was separated from background fluorescence using the lower threshold value (blue). The upper threshold value (green) was used to exclude necrotic areas of the tumor. 

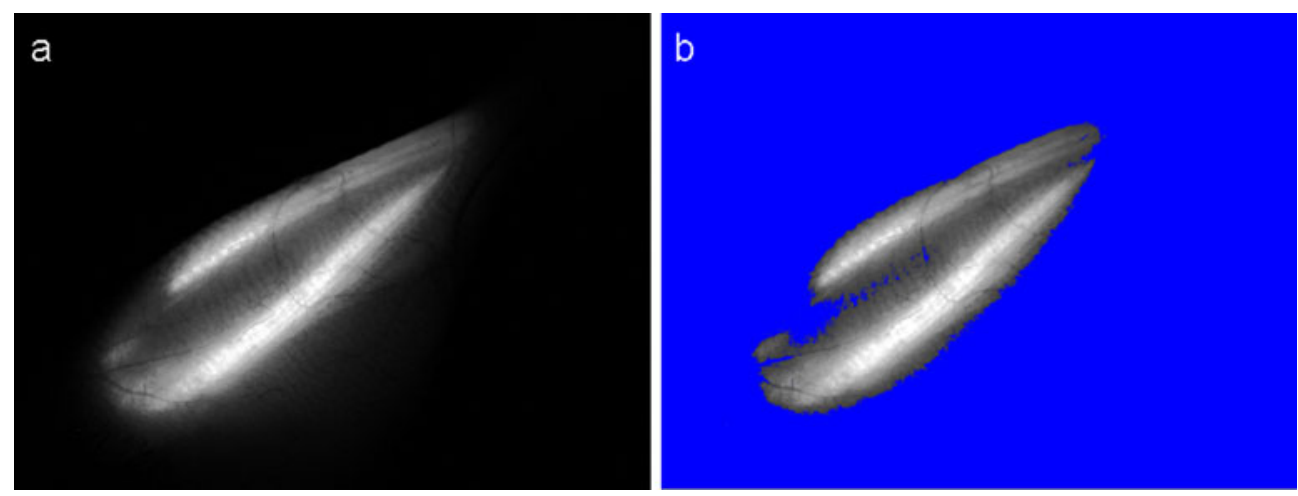

Fig. 2. a Fluorescent image of the muscle. b Fluorescence of muscle fibers was separated from the non-transfected region of the leg and background fluorescence by adjusting the lower threshold value (b/ue).

the fourth day post-treatment for tumors and on the fifth day for muscles with a factor of increase 1.35 and 1.18 , respectively (Fig. 6).

\section{Discussion}

The results of our study demonstrate that the CMV promoter cannot be considered a constitutive promoter. We show that it is silenced with time and that it can be (re)activated after treatment with a demethylating agent. We also showed that the CMV promoter can be induced by treatment with IR and CDDP in stably transformed cell lines in vitro and in vivo and after transient delivery to muscle. Furthermore, we demonstrated that non-invasive fluorescence imaging is an appropriate and convenient method to monitor the activity of the promoter in vivo.

Sustained and well-defined or controllable expression levels of the transgene are desired characteristics of mammalian gene expression vectors used for research or therapeutic purposes. Since expression levels of transgenes are dependent on the promoter, proper choice of promoter linked to the gene of interest is essential for the success of these vectors, especially if they are going to be used in human gene therapy. The CMV promoter is one of the most commonly used promoters for expression of transgenes in mammalian cells. Despite the fact that many reports demonstrated that its transcriptional activity can be changed under specific conditions, it is still used as a constitutive promoter in many in vitro and in vivo studies and even in clinical gene therapy trials [24].

The basis of our experiments was construction of different systems (stably transfected cell lines, stably transfected tumors, and transiently transfected muscles) in which expression of the GFP reporter gene was regulated either by the inducible p21 or CMV promoter. These systems allowed us to follow promoter activity simply by quantifying fluorescence intensities either by fluorescence microscopy and fluorometer in vitro or by non-invasive in vivo imaging using a fluorescence stereomicroscope, assuming that fluorescence of the cells is proportional to promoter-driven expression.

For construction of stably transfected tumor cell lines, two histologically different murine cell lines were used: mammary adenocarcinoma and fibrosarcoma. At the end of the culturing period in the selection agent, the percentage of cells expressing GFP differed between the cell lines. The observed difference can be ascribed to the cellular specificity of the CMV promoter. Namely, studies demonstrated that the CMV promoter exhibits cellular specificity and that it is active only in cell types which are naturally infected by the virus $[11,25,26]$. These studies were done on transgenic mice expressing a reporter gene under the control of CMV,
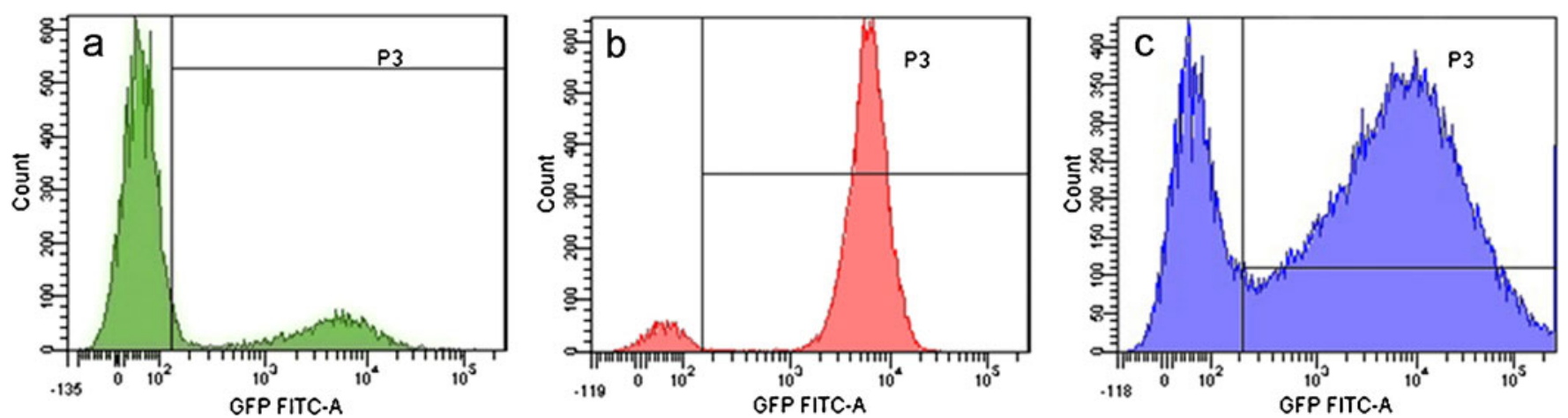

Fig. 3. Histograms of GFP fluorescence in a LPB EGFP, $\mathbf{b}$ LPB p21-EGFP, and $\mathbf{c}$ TS/A EGFP cells. 
a
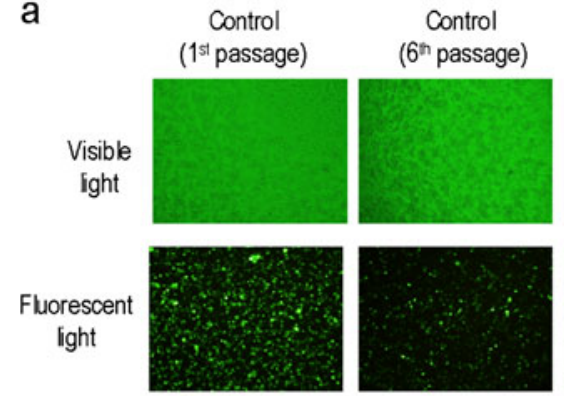

Fluorescence intensity surface plot
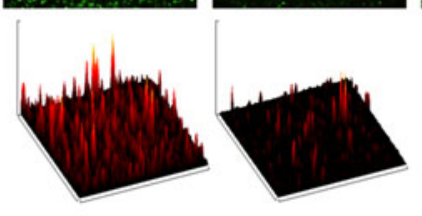

C

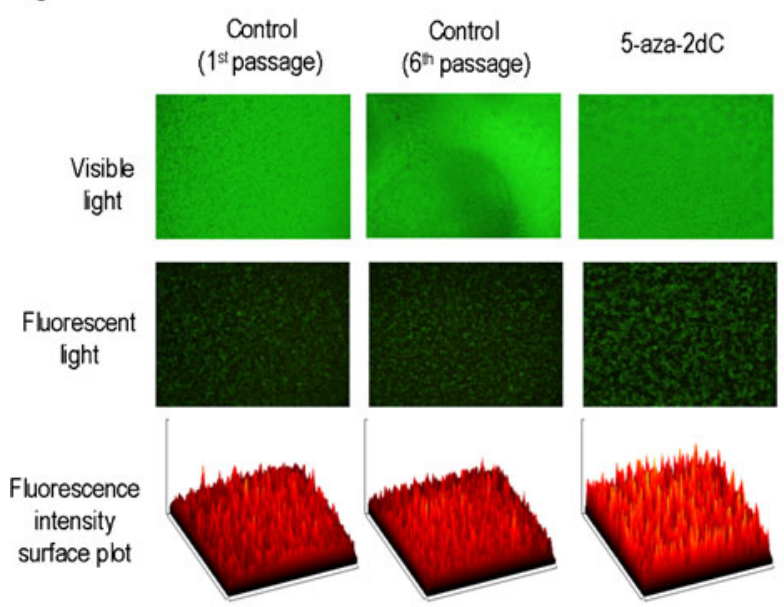

b

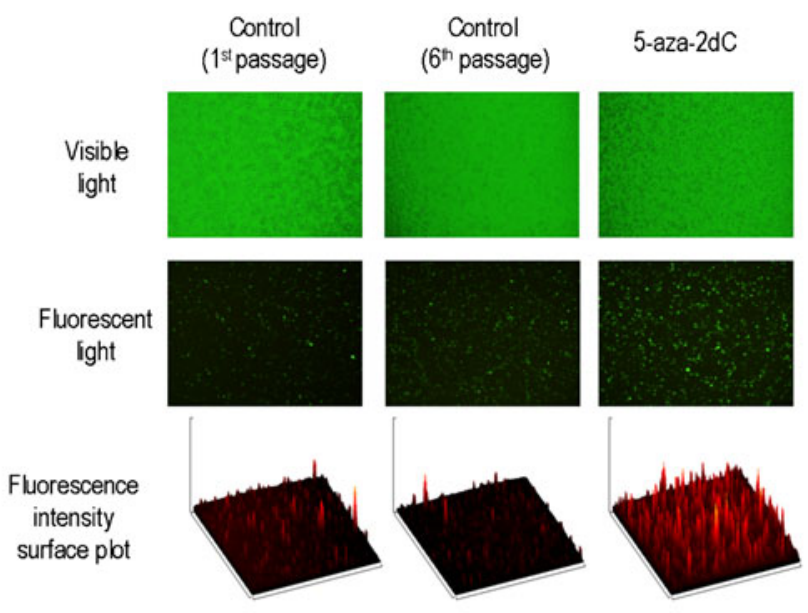

d

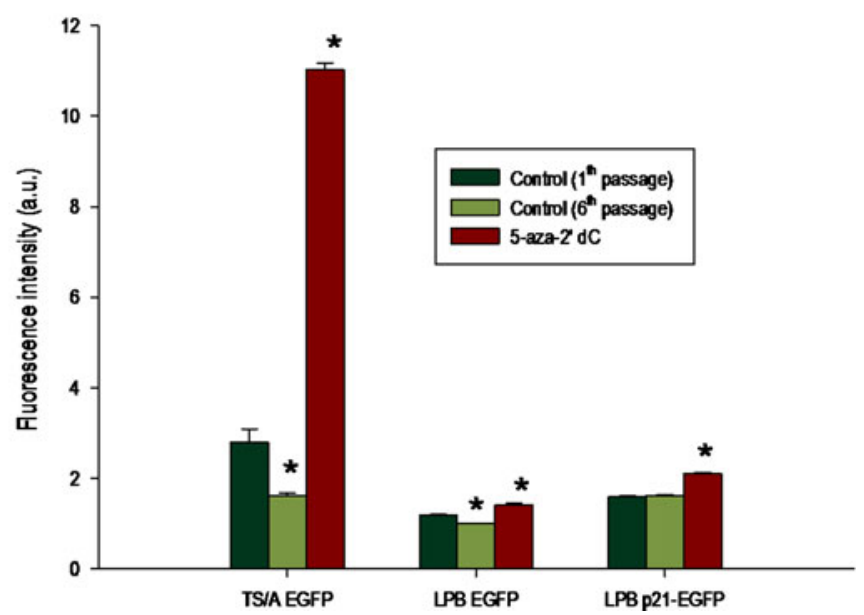

Fig. 4. Visible and fluorescence images of a TS/A EGFP, $\mathbf{b}$ LPB EGFP, and $\mathbf{c}$ LPB p21-EGFP cells. $\mathbf{d}$ Influence of treatment with 5-aza-2dC on CMV promoter reactivation. All data were pooled from three independent experiments performed in 12 replicates. Results are expressed as the mean \pm SE $\left({ }^{*} P<0.05\right)$.

thus, in non-transformed non-cancer cells. In our case, TS/A, which is murine adenocarcinoma of the mammary glands, and LPB, which is murine fibrosarcoma, cell lines were used. Although the cell lines in our study were transformed cancer cell lines, they are derived from two different cell types. Therefore, it can be speculated that differences in the percentage of stably transfected cells were due to the inherent differences in these cell lines with regard to susceptibility to infection with CMV.

Another observation during the preparation of stable cell lines was that initial high fluorescence intensity decreased after every round of replication in cell lines with the CMV promoter, but not in the cell line with the p21 promoter. Several mechanisms for the transient expression of the delivered transgene have been described in the literature, such as loss of vector DNA from the transduced cells and transcriptional inactivation of the promoter [27, 28]. In our case, the loss of vector DNA could not be a major limiting factor for sustained transgene expression, since "stably" transfected cells were resistant to increasing concentrations of geneticin, meaning that they expressed the selective marker encoded on the plasmid, which demonstrates that the plasmid DNA is present in the cells. In addition, results of our previous study, showing that long-term transgene expression can be maintained even after transient transfection in muscle, support the argument that the major cause for the observed loss of transgene expression was promoter inactivation rather than the loss of plasmid [23].

DNA methylation of the promoter is the most frequent justification for inactivation of the promoter [1, 2, 4, 5, 29]. The process of promoter methylation is mediated by a class of enzymes that covalently link a methyl group to the promoter region of the gene leading to modification of accessibility of transcriptional factors to the promoter. To test if the observed decrease in reporter gene expression in our study was due to methylation of the CMV promoter, freshly thawed cells were first passaged for 1 month to allow methylation of DNA to take place and then treated with the demethylation agent 5 -aza-2dC, which is one of the best characterized drugs used for reactivation of methylated 
a
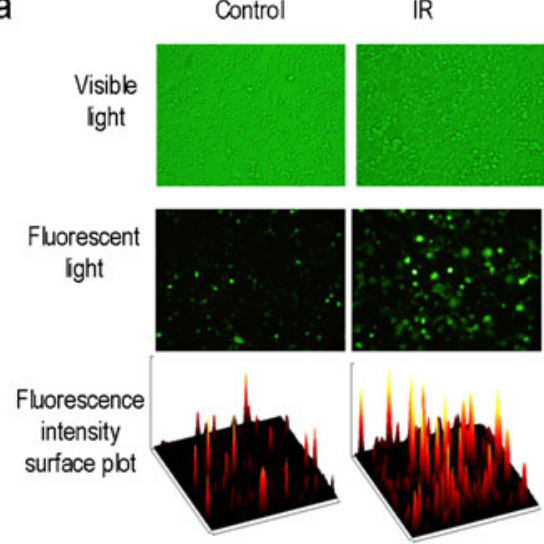

C

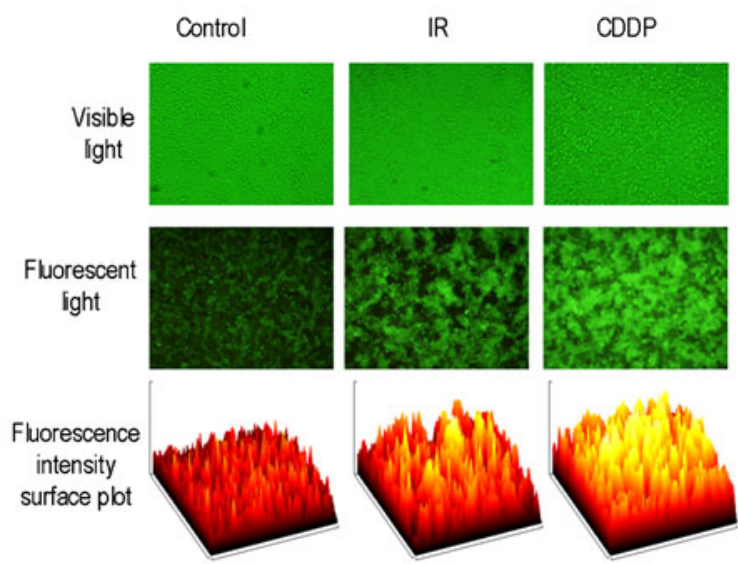

b

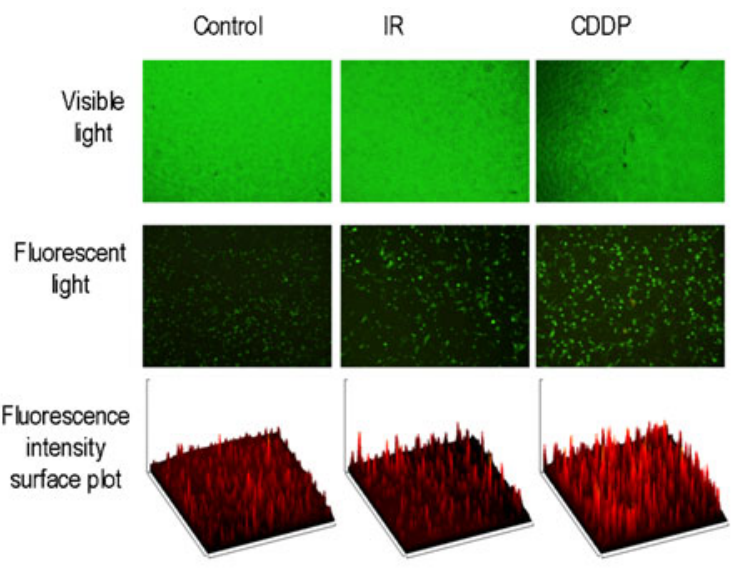

d

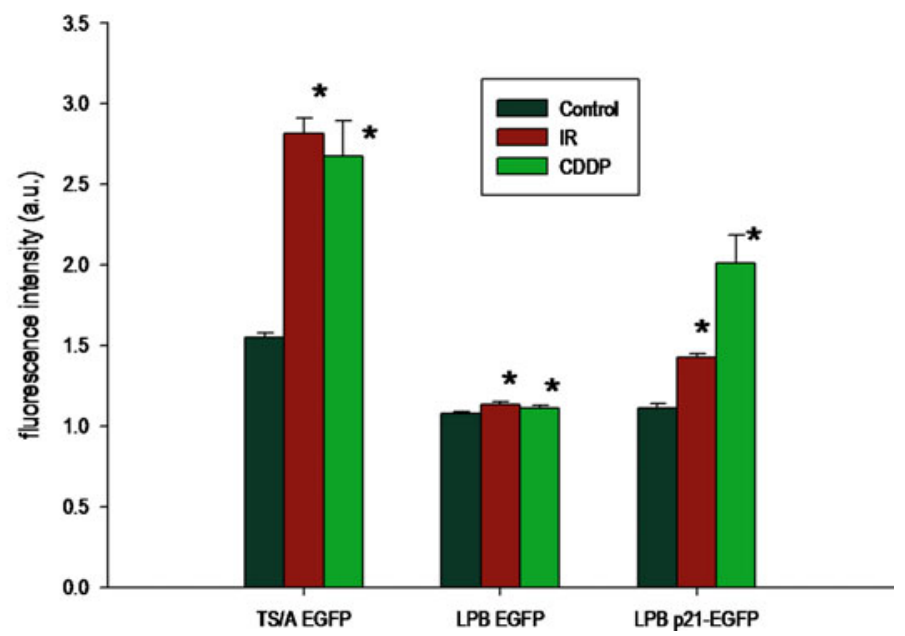

Fig. 5. Visible and fluorescence images of a TS/A EGFP, $\mathbf{b}$ LPB EGFP, and $\mathbf{c}$ LPB p21-EGFP cells. $\mathbf{d}$ IR and CDDP induced upregulation of the CMV promoter. All data were pooled from three independent experiments performed in 12 replicates. Results are expressed as the mean \pm SE $\left({ }^{\star} P<0.05\right)$.

promoters [2, 30-32]. The fluorescence intensity of the first passage of freshly thawed cells was taken as the reference for the unmethylated state. After the treatment, an obvious increase in fluorescence was obtained, which was more pronounced in the TS/A EGFP cell line $(6.9 \times)$ than in the LPB EGFP cell line $(1.4 \times)$. Similar to our results, reactivation of CMV-driven reporter gene expression after treatment with 5 -aza-2dC was reported in a study using lipofection to stably transfect a human glioblastoma cell line [3].

Interestingly, in our study, the increase in fluorescence after (re)activation was higher than would be expected from the reference (fluorescence intensity of the cells from the first passage), especially in the TS/A cell line, indicating that some other mechanisms were involved in the observed (re) activation. One of them could be upregulation induced by cytotoxic stress. Namely, in addition to its demethylating function, 5-aza-2-dC has also been shown to possess significant cytotoxic and anticancer activity. In fact, it was originally developed as an anticancer agent and was as such used in many preclinical and clinical trials [33]. There are two lines of evidence supporting the involvement of stress in the observed upregulation in our study. The first is that some upregulation occurred also after treatment with 5 -aza-2dC in the control group with the p21 promoter. These results are in accordance with other studies [34-38], where cytotoxicity of 5-aza-2dC was linked to its ability to induce DNA damage, leading to activation of the $p 53$ and consequently $p 21$ pathways. The next line of evidence supporting the involvement of stress is upregulation of the CMV promoter which was obtained after exposure of cells to IR and treatment with CDDP. Therefore, the increase in fluorescence intensity above the reference value in the two stably transfected cell lines with the CMV promoter is most probably the sum of demethylation of the CMV promoter and upregulation of the CMV promoter due to the cytotoxic action of 5-aza-2dC. However, to fully validate the involvement of DNA methylation in the (re)activation of the CMV promoter, further experiments, such as methylation-sensitive restriction enzyme analysis, should be performed [7].

To test whether inactivation of the CMV promoter also occurs in in vivo conditions, in vitro results were verified by in vivo imaging in a tumor-bearing mouse model. We only used the TS/A EGFP tumor model since the reporter gene expression in the LPB EGFP cell line was too low and could 
a

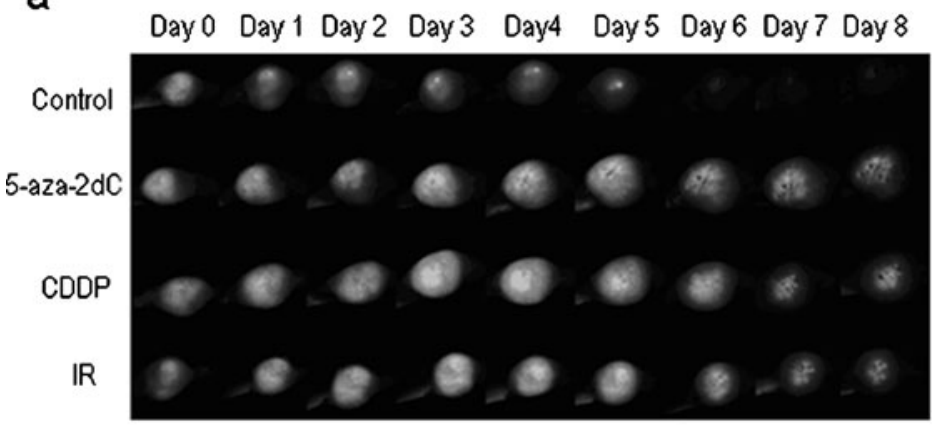

C

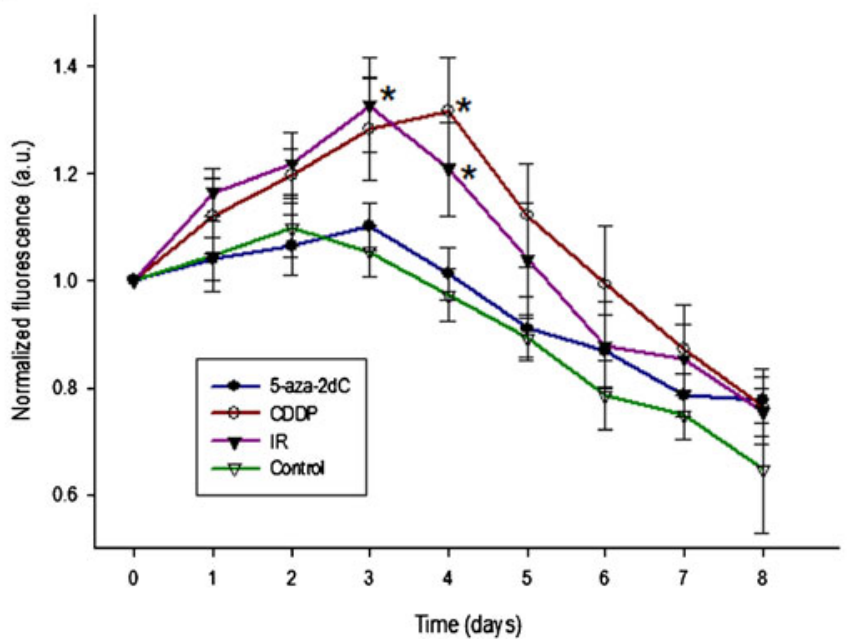

b

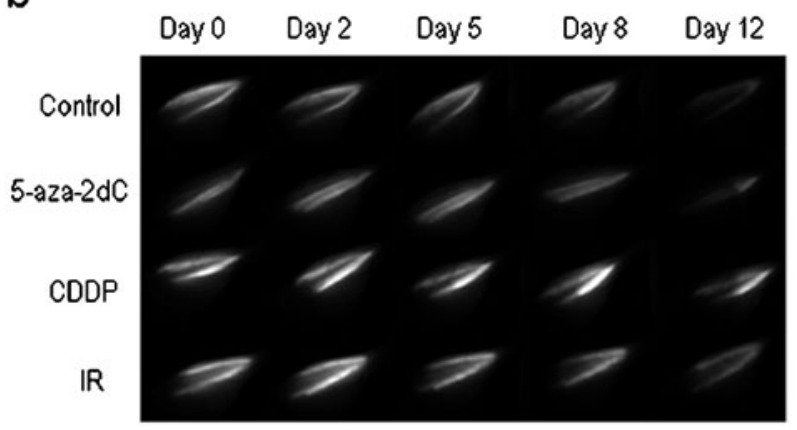

Fig. 6. Fluorescence images of a TS/A EGFP tumors, and $\mathbf{b}$ transiently transfected muscles after different treatments. Influence of treatment with 5-aza-2dC, IR, and CDDP on CMV activity in c TS/A-EGFP tumor model, and $\mathbf{d}$ transiently transfected muscles. Results are expressed as the mean \pm SE $\left({ }^{*} P<0.05\right)$.

not be detected transcutaneously due to technical limitations of the fluorescence stereomicroscope. In the TS/A EGFP tumors, the effects of treatment with 5 -aza- $2 \mathrm{dC}$ were not as obvious as in vitro; only the trend of increased fluorescence intensity was indicated. The reason for the observed difference between the in vitro and in vivo results is most probably due to the fast growth of tumors which limit the duration of the experiment ( 10 days compared to 1 month used in in vitro experiments) and consequently the time needed for methylation to occur. This observation is also supported by the fact that the trend was more pronounced toward the end of the experiment. To our knowledge, our study is the first study dealing with methylation of the CMV promoter in tumors. Other studies dealing with methylation of CMV were done in normal tissues and their results are inconsistent. Association of transcriptional silencing with methylation of the CMV promoter was demonstrated in rat muscles using adenoviral gene delivery [1]. On the other hand, the results of other studies showed that methylation is not responsible for transient transgene expression in vivo after adenovirus gene transfer in the mouse liver [7] and after non-viral vectors delivery in the mouse lung [6]. In these reports, promoter attenuation due to inflammatory processes was proposed as an alternative mechanism for the decrease in transgene expression.

To confirm that methylation of the CMV promoter is associated with transcriptional silencing in muscle also after non-viral gene delivery methods, as was demonstrated for viral delivery [1], additional experiments were performed in transiently transfected muscles carrying CMV promoterdriven GFP. Treatment with 5 -aza-2dC was performed 1 month after transfection, when the fluorescence intensity of GFP reached $\sim 50 \%$ of the highest value [23], and we could anticipate that there was enough time for methylation to occur. After treatment, fluorescence intensity was significantly increased compared to the control. Therefore, our results indicate that methylation could be responsible for the observed increase in fluorescence intensity after transient transfection in muscle and support the results obtained in rat muscle after adenoviral-mediated transfection [1].

The next part of our study dealt with the stress-induced upregulation of the CMV promoter. We used the same systems as for the methylation tests, but instead of treatment with 5-aza-2dC, we utilized two standard anticancer treat- 
ments: local IR and systemic treatment with the chemotherapeutic agent CDDP that cause DNA damage. To avoid the effect of methylation, freshly thawed cells were used in the experiments. The exposure of cells to IR and CDDP induced a significant increase in fluorescence intensity compared to untreated controls. However, the magnitude of this increase varied among the cell lines, being high in TS/A EGFP cells and low in LPB EGFP cells. Furthermore, the magnitude of induction was similar to that obtained with the inducible p21 promoter. Therefore, in our study in LPB cells, the CMV promoter was as inducible as the known inducible p21 promoter [18, 19]. Similar observations have been previously demonstrated for different cell lines using different reporter genes and different delivery systems $[13,14$, 39]. For example, the chemotherapeutic agent doxorubicin was reported to upregulate CMV promoter-driven reporter gene (luciferase) expression in stably transfected cell lines [14, 39] and after transient transfection using lipofectamine [14] and an adenoviral vector [17]. IR (10 Gy) was also reported to upregulate a CMV promoter-driven reporter gene in cells transfected using electroporation [13].

In our study, significant upregulation of the CMV promoter with IR and CDDP was also demonstrated in vivo in tumors as well as in transiently transfected muscles. Similar to our study, luminescence-based whole body imaging showed upregulation of the CMV promoter between 4 and 10 days after treatment of animals with doxorubicin [14]. This upregulation of the CMV promoter is of special importance when gene therapy is intended to be combined with other standard cancer treatments, such as radiotherapy and chemotherapy. Increased expression of the therapeutic protein and consequently an increased therapeutic effect can occur after treatment of patients with standard therapy. Non-invasive imaging methods should be taken into consideration when gene therapy is combined with treatments that upregulate the promoter used in the gene therapy vector, as non-invasive imaging can give the exact time dependence of the activity of the promoter and therefore optimal planning for the combination of therapies can be achieved. Fluorescence imaging has in this case, an advantage over luminescence imaging, since no additional substrate is required for visualization of transgene expression and can be safely used over a prolonged period of time, although with bioluminescence imaging lower levels of the reporter gene can be detected [15].

\section{Conclusions}

Our study demonstrated that the CMV promoter can be upregulated by different treatments. Observed alterations in the activity of the CMV promoter limit the usefulness of this widely used promoter as a constitutive promoter and highlight the importance of proper choice of promoter linked to the gene of interest for success of gene therapy as well as basic research. On the other hand, inducibility of CMV promoters can be beneficially used in gene therapy when combined with standard cancer treatment, such as radiotherapy and chemotherapy. Non-invasive imaging methods have great potential for development of these treatment combinations that will have an enhanced antitumor effect at the same level of normal tissue damage.

Acknowledgment. The authors acknowledge the financial support of the state budget through the Slovenian Research Agency (P3-0003: J3-9580, J3-0485).

Open Access. This article is distributed under the terms of the Creative Commons Attribution Noncommercial License which permits any noncommercial use, distribution, and reproduction in any medium, provided the original author(s) and source are credited.

\section{References}

1. Brooks AR, Harkins RN, Wang PY, et al. (2004) Transcriptional silencing is associated with extensive methylation of the CMV promoter following adenoviral gene delivery to muscle. J Gene Med 6:395-404

2. Escher G, Hoang A, Georges S, et al. (2005) Demethylation using the epigenetic modifier, 5-azacytidine, increases the efficiency of transient transfection of macrophages. J Lipid Res 46:356-365

3. Grassi G, Maccaroni P, Kaiser H, et al. (2003) Inhibitors of DNA methylation and histone deacetylation activate cytomegalovirus promoter-controlled reporter gene expression in human glioblastoma cell line U87. Carcinogenesis 24:1625-1635

4. Hong K, Sherley J, Lauffenburger DA (2001) Methylation of episomal plasmids as a barrier to transient gene expression via a synthetic delivery vector. Biomol Eng 18:185-192

5. Prosch S, Stein J, Staak K, et al. (1996) Inactivation of the very strong HCMV immediate early promoter by DNA CpG methylation in vitro. Biol Chem 377:195-201

6. Pringle IA, Raman S, Sharp WW, et al. (2005) Detection of plasmid DNA vectors following gene transfer to the murine airways. Gene Ther 12:1206-1214

7. Loser P, Jennings GS, Strauss M, Sandig V (1998) Reactivation of the previously silenced cytomegalovirus major immediate-early promoter in the mouse liver: involvement of NF kappa B. J Virol 72:180-190

8. Lee Y, Sohn WJ, Kim DS, Kwon HJ (2004) NF-kappa B- and c-Jundependent regulation of human cytomegalovirus immediate-early gene enhancer/promoter in response to lipopolysaccharide and bacterial CpGoligodeoxynucleotides in macrophage cell line RAW 264.7. Eur J Biochem 271:1094-1105

9. Ramanathan M, Hasko G, Leibovich SJ (2005) Analysis of signal transduction pathways in macrophages using expression vectors with CMV promoters: a cautionary tale. Inflammation 29:94-102

10. Simpson AJ, Cunningham GA, Porteous DJ, Haslett C, Sallenave JM (2001) Regulation of adenovirus-mediated elafin transgene expression by bacterial lipopolysaccharide. Hum Gene Ther 12:1395-1406

11. Stamminger T, Fickenscher H, Fleckenstein B (1990) Cell type-specific induction of the major immediate early enhancer of human cytomegalovirus by cyclic-amp. J Gen Virol 71:105-113

12. Bruening W, Giasson B, Mushynski W, Durham HD (1998) Activation of stress-activated MAP protein kinases up-regulates expression of transgenes driven by the cytomegalovirus immediate/early promoter. Nucleic Acids Res 26:486-489

13. Egami T, Huchida K, Mizumoto K, et al. (2008) Radiation enhances adenoviral gene therapy in pancreatic cancer via activation of cytomegalovirus promoter and increased adenovirus uptake. Clin Cancer Res 14:1859-1867

14. Svensson RU, Barnes JM, Rokhlin OW, Cohen NB, Henry MD (2007) Chemotherapeutic agents up-regulate the cytomegalovirus promoter: implications for bioluminescence Imaging of tumor response to therapy. Cance Res 67:10445-10454

15. Raty JK, Liimatainen T, Kaikkonen MU, et al. (2007) Non-invasive imaging in gene therapy. Mol Ther 15:2052

16. Kang JH, Chung JK (2008) Molecular-genetic imaging based on reporter gene expression. J Nuc Med 49:164S-179S

17. Kim SJ, Varghese TK, Zhang Z, et al. (2005) Renal ischemia/reperfusion injury activates the enhancer domain of the human cytomegalovirus major immediate early promoter. Am J Transplant 5:1606-1613 
18. Eldeiry WS, Tokino T, Velculescu VE, et al. (1993) Waf1, a potential mediator of P53 tumor suppression. Cell 75:817-825

19. Harada K, Ogden GR (2000) An overview of the cell cycle arrest protein, p21(WAF1). Oral Oncol 36:3-7

20. Nanni P, Degiovanni C, Lollini PL, Nicoletti G, Prodi G (1983) Ts/A a new metastasizing cell-line from $\mathrm{A} \mathrm{Balb/C}$ spontaneous mammary adenocarcinoma. Clin Exp Metas 1:373-380

21. Belehradek J, Barski G, Thonier M (1972) Evolution of cell-mediated antitumor immunity in mice bearing a syngeneic chemically induced tumor. Influence of tumor growth, surgical removal and treatment with irradiated cells. Int J Cancer 9:461-469

22. Teissie J, Escoffre JM, Rols MP, Golzio M (2008) Time dependence of electric field effects on cell membranes. A review for a critical selection of pulse duration for therapeutical applications. Radiol Oncol 42:196-206

23. Tevz G, Pavlin D, Kamensek U, et al. (2008) Gene electrotransfer into murine skeletal muscle: a systematic analysis of parameters for longterm gene expression. Technol Cancer Res T 7:91-101

24. O'Malley BW, Li DQ, McQuone SJ, Ralston R (2005) Combination nonviral interleukin-2 gene immunotherapy for head and neck cancer: From bench top to bedside. Laryngoscope 115:391-404

25. Furth PA, Hennighausen L, Baker C, Beatty B, Woychick R (1991) The variability in activity of the universally expressed human cytomegalovirus immediate early gene-1 enhancer promoter in transgenic mice. Nucleic Acids Res 19:6205-6208

26. Baskar JF, Smith PP, Nilaver G, et al. (1996) The enhancer domain of the human cytomegalovirus major immediate-early promoter determines cell type-specific expression in transgenic mice. J Virol 70:3207-3214

27. Yew NS, Przybylska M, Ziegler RJ, Liu DP, Cheng SH (2001) High and sustained transgene expression in vivo from plasmid vectors containing a hybrid ubiquitin promoter. Mol Ther 4:75-82

28. Ritter T, Brandt C, Prosch S, et al. (2000) Stimulatory and inhibitory action of cytokines on the regulation of hCMV-IE promoter activity in human endothelial cells. Cytokine 12:1163-1170
29. Vaissiere T, Sawan C, Herceg Z (2008) Epigenetic interplay between histone modifications and DNA methylation in gene silencing. Mutat Res-Rev Mutat 659:40-48

30. Jaenisch R, Schnieke A, Harbers K (1985) Treatment of mice with 5azacytidine efficiently activates silent retroviral genomes in different tissues. P Natl Acad Sci USA 82:1451-1455

31. Krishnan M, Park JM, Cao F, et al. (2005) Effects of epigenetic modulation on reporter gene expression: implications for stem cell imaging. Faseb J 19:106

32. Di Ianni M, Terenzi A, Perruccio K, et al. (1999) 5-Azacytidine prevents transgene methylation in vivo. Gene Ther 6:703-707

33. Momparler RL (2005) Epigenetic therapy of cancer with 5-aza-2 'deoxycytidine (decitabine). Sem Oncol 32:443-451

34. Deng T, Zhang Y (2009) Possible involvement of activation of P53/P21 and demethylation of RUNX 3 in the cytotoxicity against Lovo cells induced by 5-Aza-2'-deoxycytidine. Life Sci 84:311-320

35. Karpf AR, Moore BE, Ririe TO, Jones DA (2001) Activation of the p53 DNA damage response pathway after inhibition of DNA methyltransferase by 5-aza-2'-deoxycytidine. Mol Pharmacol 59:751-757

36. Pulukuri SMK, Rao JS (2005) Activation of p53/p21(Wafl/Cip1) pathway by 5 -aza-2'-deoxycytidine inhibits cell proliferation, induces pro-apoptotic genes and mitogen-activated protein kinases in human prostate cancer cells. Int J Oncol 26:863-871

37. Zhu WG, Hileman T, Ke Y, et al. (2004) 5-Aza-2'-deoxycytidine activates the $\mathrm{p} 53 / \mathrm{p} 21$ (Waf1/Cip1) pathway to inhibit cell proliferation. J Biol Chem 279:15161-15166

38. Schneider-Stock R, ab-Assef M, Rohrbeck A, et al. (2005) 5-Azacytidine is a potent inhibitor of DNA methyltransferase $3 \mathrm{a}$ and induces apoptosis in HCT-116 colon cancer cells via Gadd45-and p53-dependent mechanisms. J Pharmacol Exp Ther 312:525-536

39. Kim KI, Kang JH, Chung JK, et al. (2007) Doxorubicin enhances the expression of transgene under control of the CMV promoter in anaplastic thyroid carcinoma cells. J Nucl Med 48:1553-1561 